\title{
Mathematical Modeling of Atmospheric Flow and Computation of Convex Envelopes
}

\author{
A. Caboussat * \\ Department of Mathematics, University of Houston, Houston, Texas 77204-3008, USA
}

\begin{abstract}
Atmospheric flow equations govern the time evolution of chemical concentrations in the atmosphere. When considering gas and particle phases, the underlying partial differential equations involve advection and diffusion operators, coagulation effects, and evaporation and condensation phenomena between the aerosol particles and the gas phase. Operator splitting techniques are generally used in global air quality models. When considering organic aerosol particles, the modeling of the thermodynamic equilibrium of each particle leads to the determination of the convex envelope of the energy function. Two strategies are proposed to address the computation of convex envelopes. The first one is based on a primal-dual interior-point method, while the second one relies on a variational formulation, an appropriate augmented Lagrangian, an Uzawa iterative algorithm, and finite element techniques. Numerical experiments are presented for chemical systems of atmospheric interest, in order to compute convex envelopes in various space dimensions.
\end{abstract}

Key words: atmospheric flow, air quality, convex envelopes, interior-point method, variational problem, augmented Lagrangian

AMS subject classification: 90C51, 65N30, 65K10, 86A10, 49M15

\section{Introduction}

Atmospheric flow and air pollution models are widely used in many environmental applications $[23,30,31]$. All these applications are usually based on the so-called chemistry transport models, which govern the time evolution of the chemical components in the atmosphere. These components in the atmosphere exist in various phases, namely the gas phase and the particle phase that consists

*Corresponding author. E-mail: caboussat@math.uh.edu 
of atmospheric aerosol particles of different sizes. Aerosol particles are of paramount importance in the modeling of atmospheric pollution and air quality $[18,28,29]$ as they have numerous effects: besides influencing human health, visibility in urban and regional areas, or formation of acid rain, these particles strongly alter the earth's radiation balance, and the cloud or ozone formation.

The chemical components in the atmosphere undergo a wide range of physical and chemical transformations. These phenomena represent macroscopic effects (such as advection and diffusion processes), as well as microscopic effects (such as nucleation, coagulation, condensation and evaporation). The partial differential equations governing the gas and particle phase respectively are coupled through the mass transfers between the two phases. At the numerical level, the use of operator splitting in the solution of atmospheric flow equations separates the solution of pollution dynamics into four key operators: the advection-diffusion operator, the chemical dynamics, the emissions and deposition processes, and the aerosol transport and formation [10, 21].

The aerosol transport and formation step includes the description of the chemical composition and size distribution of aerosols $[32,33]$. These particles are generally a mixture of inorganic and organic components and water, and their size goes over a wide range of scales. For given atmospheric conditions, volatile species are distributed between the gas and aerosol phases according to fluxes that depend on the thermodynamic equilibrium inside the particle phase.

A great deal of work has been carried out on the development of thermodynamic models of atmospheric aerosols. We focus here on organic particles $[1,4,6]$ and, within the operator splitting algorithm, address the global minimization of their internal energy at the microscopic level. Via a suitable transformation, we link this thermodynamic equilibrium problem with the computation of convex envelopes $[1,4,20]$, and discuss two numerical methods for their computation: the first one is based on an primal-dual interior-point method, coupled with an active set/Newton method $[4,5,13,17]$; the second one has a variational flavor, and relies on an appropriate augmented Lagrangian $[14,16]$, a Uzawa-type iterative algorithm, and a finite element discretization similar to the one presented in $[7,8,15]$.

This article is organized as follows: in Section 2., we present the system of partial differential equations governing the atmospheric flow. Section 3. focuses on the microscopic model obtained for the interaction between gas and particle phases after space discretization and a suitable operator splitting. Section 4. details the link with the computation of convex envelopes, and, in Sections 5. and 6., we present two methods for the calculations of such envelopes, based on an interior-point method and a variational problem respectively. Numerical results are exhibited in Section 7..

\section{Modeling of Atmospheric Flow}

The atmosphere is composed of many chemical components that undergo physical and chemical transformations. In particular, the chemical components exist in various phases, namely the gas phase, and the particle phase, composed by aerosol particles. Let us denote by $\overline{\mathbf{b}}=\left(\bar{b}_{i}\right)_{i=1}^{s}$, and $\overline{\mathbf{c}}=\left(\bar{c}_{i}\right)_{i=1}^{s}$, the concentrations of chemical components in the particle phase and in the gas phase respectively. Both concentration vectors are functions of space $(\mathbf{x})$ and time $(t)$ variables in a global three-dimensional model. In addition, the concentrations in the particle phase $\overline{\mathbf{b}}$ are functions of the 
size of the particle. In the remainder of this article, let us assume that these particles are spherical and denote by $R$ the radius of such particles; the concentrations $\bar{b}$ will therefore depend on $R$. The coagulation and condensation/evaporation kernels depend directly on this parametrization choice.

The continuous general dynamic equations governing these concentrations are the following, for $i=1, \ldots, s$ (see, e.g., $[18,28]$ ). This system can also include scavenging and deposition processes. In order not to complicate the notation, these effects are not included here.

$$
\begin{gathered}
\frac{\partial}{\partial t} \bar{b}_{i}(R, \mathbf{x}, t)+\mathbf{u}(\mathbf{x}, t) \cdot \nabla_{\mathbf{x}} \bar{b}_{i}(R, \mathbf{x}, t)-\nabla_{\mathbf{x}} \cdot\left(\mathbf{K}(\mathbf{x}, t) \nabla_{\mathbf{x}} \bar{b}_{i}(R, \mathbf{x}, t)\right) \\
-\frac{I_{i}(R, \mathbf{x}, t)}{R} \bar{b}_{s}(R, \mathbf{x}, t)+\frac{\partial}{\partial R}\left(I_{s}(R, \mathbf{x}, t) \bar{b}_{i}(R, \mathbf{x}, t)\right)=S_{i}(R, \mathbf{x}, t)+ \\
\int_{0}^{R} \beta\left(R-R^{\prime}, R^{\prime}\right) \frac{\bar{b}_{s}\left(R-R^{\prime}, \mathbf{x}, t\right)}{R-R^{\prime}} \bar{b}_{i}\left(R^{\prime}, \mathbf{x}, t\right) d R^{\prime} \\
-\bar{b}_{i}(R, \mathbf{x}, t) \int_{0}^{\infty} \beta\left(R, R^{\prime}\right) \frac{\bar{b}_{s}\left(R^{\prime}, \mathbf{x}, t\right)}{R^{\prime}} d R^{\prime} \\
\frac{\partial}{\partial t} \bar{c}_{i}(\mathbf{x}, t)+\mathbf{u}(\mathbf{x}, t) \cdot \nabla_{\mathbf{x}} \bar{c}_{i}(\mathbf{x}, t)-\nabla_{\mathbf{x}} \cdot\left(\mathbf{K}(\mathbf{x}, t) \nabla_{\mathbf{x}} \bar{c}_{i}(\mathbf{x}, t)\right)+ \\
\int_{0}^{\infty} \frac{\bar{b}_{s}\left(R^{\prime}, \mathbf{x}, t\right)}{R^{\prime}} I_{i}\left(R^{\prime}, \mathbf{x}, t\right) d R^{\prime}=E_{i}(\mathbf{x}, t)
\end{gathered}
$$

where $R$ is the variable describing the size of the particle (radius of a spherical particle), $(\mathbf{x}, t)$ are the space and time variables. Here $\mathbf{u}$ is a given advection field (e.g. wind velocity), $\mathbf{K}$ is a diffusion tensor, $S_{i}$ and $E_{i}$ are the sources (emissions of chemicals), and $\beta\left(R, R^{\prime}\right)$ is a probability of coagulation between particles of sizes $R$ and $R^{\prime}$. The two integrals describes the coagulation processes that allow particles of various sizes to coalesce or split. The quantity $\bar{b}_{s}(R, \mathbf{x}, t)$ is defined as $\bar{b}_{s}(R, \mathbf{x}, t)=\sum_{i=1}^{s} \bar{b}_{i}(R, \mathbf{x}, t)$. The coupling between (2.1) and (2.2) is a result of condensation and evaporation processes, described by

$$
I_{i}(R, \mathbf{x}, t)=h_{i}(R)\left(\bar{c}_{i}(\mathbf{x}, t)-\eta(R) \frac{1}{\mathcal{R} T(\mathbf{x}, t)} p_{i}^{\text {equil }}(\mathbf{b}(R, \mathbf{x}, t))\right),
$$

with $I_{s}(R, \mathbf{x}, t)=\sum_{i=1}^{s} I_{i}(R, \mathbf{x}, t)$. Here $\eta(R)$ is the Kelvin effect for a particle of size/radius $R$, that takes into account the effects due to the curvature of the interface of the particle, $h_{i}(R)$ is the molecular transfer coefficient that regulates the reaction speed of the species $i$ between gas and particle, $\mathcal{R}$ is the ideal gas constant $\left(\mathcal{R}=8.20574587 \cdot 10^{-5}\left[\mathrm{~m}^{3} \mathrm{~atm} \mathrm{~K}^{-1} \mathrm{~mol}^{-1}\right]\right)$, $T$ is the temperature, and $p_{i}^{\text {equil }}(\mathbf{b})$ is the fugacity of the gas species $i$ corresponding to the particle composition $\mathbf{b}$. The fugacity corresponds to a pressure/concentration at the surface of the particle, and is the result of the microscopic modeling of the chemical components in the particle phase described hereafter. The flux (2.3) describes the equilibrium of concentrations at the interface of the particle. 


\section{Microscopic Modeling of Aerosol Particles}

In three-dimensional global air quality algorithms, the physical phenomena in the system of partial differential equations are decoupled with a time splitting scheme and discretized in space. Besides the macroscopic operators (advection and diffusion), the microscopic processes involve phase transformation and chemical reactions [24, 25, 26]. The two main processes involving the particle phase are the thermodynamic equilibrium [4,6] inside the particle and the mass transfer between the gas phase and the particle phase [1,9,20].

We assume now that there is one single particle of size $R$ in the system. Discretizing (2.1) (2.2) in space, evaluating the system at this particular radius $R$, and using an operator splitting algorithm, the sub-system corresponding to the mass transfer and thermodynamic equilibrium of this single aerosol particle consists of the equations:

$$
\begin{aligned}
\frac{d}{d t} c_{i}(t)=-h_{i}(R)\left(c_{i}(t)-\eta(R) \frac{1}{\mathcal{R} T(t)} p_{i}^{\text {equil }}(\mathbf{b}(t))\right), & i=1, \ldots, s, \\
\frac{d}{d t} b_{i}(t)=h_{i}(R)\left(c_{i}(t)-\eta(R) \frac{1}{\mathcal{R} T(t)} p_{i}^{\text {equil }}(\mathbf{b}(t))\right), & i=1, \ldots, s,
\end{aligned}
$$

where $\mathbf{b}=\left(b_{i}\right)_{i=1}^{s}$ and $\mathbf{c}=\left(c_{i}\right)_{i=1}^{s}$ denote the (local) concentrations of chemical components in the particle phase and in the gas phase respectively. The system (3.1) (3.2) represent the transfer between gas and particle phases. The fluxes are proportional to the difference of concentrations between the gas phase and the concentrations at the interface of the particles (fugacity). The factors $h_{i}(R)$ are the mass transfer rates of each chemical component, and are functions of the size of the particle, which influences the transfer speed (as the evolution of larger particles is actually slower).

Atmospheric particles can be categorized into different species, namely organic aerosols, inorganic aerosols, and mixtures of both (see, e.g., [2, 3]). Let us consider here organic atmospheric particles. We consider the situation (common for aerosol with only organic species) with no chemical reactions occurring in the particle phase, but only a phase separation between several liquid phases. It has been shown in $[2,4]$ that the computation of the thermodynamic equilibrium for such particles corresponds to the computation of the convex envelope of some energy function. In this article, we focus therefore on organic particles and describe numerical methods adapted to this particular type of particles. Note that (different) numerical techniques for inorganic particles have been proposed in [3].

In order to determine the fugacity of the gas species $p_{i}^{\text {equil }}(\mathbf{b})$ at each time $t$, one needs to solve a phase equilibrium problem inside the particle phase, namely to find the solution of the global optimization problem governing the thermodynamics of the aerosol particle: for a given $\mathbf{b} \in \mathbb{R}^{s}$, find $y_{\alpha} \in \mathbb{R}$ and $\mathbf{x}_{\alpha} \in \mathbb{R}^{s}$, for all $\alpha=1, \ldots, p$, satisfying

$$
\begin{array}{ll}
\min _{y_{\alpha}, \mathbf{x}_{\alpha}} & \sum_{\alpha=1}^{p} y_{\alpha} g\left(\mathbf{x}_{\alpha}\right) \\
\text { s. t. } & \sum_{\alpha=1}^{p} y_{\alpha} \mathbf{x}_{\alpha}=\mathbf{b}, \quad y_{\alpha} \geq 0, \quad \mathbf{e}_{s}^{T} \mathbf{x}_{\alpha}=1, \quad \mathbf{x}_{\alpha}>\mathbf{0}, \quad \alpha=1, \ldots, p .
\end{array}
$$


Here $p$ is the (unknown) total number of liquid phases in the particle, $y_{\alpha}$ and $\mathbf{x}_{\alpha}$ are respectively the number of moles and the mole-fraction vector in the phase $\alpha$, and $\mathbf{e}_{s}=(1, \ldots, 1)^{T} \in \mathbb{R}^{s}$. The function $g \in \mathcal{C}^{\infty}\left(\mathbb{R}_{++}^{s}\right) \cap \mathcal{C}^{0}\left(\mathbb{R}_{+}^{s}\right)$ is the molar Gibbs free energy function [4] (where $\mathbb{R}_{++}$ denotes the set of positive real numbers). This function is homogeneous of degree one, non convex, nonlinear and such that $\lim _{x_{i} \rightarrow 0} \frac{\partial g}{\partial x_{i}}=-\infty, i=1, \ldots, s$, and $\mathbf{x}^{T} \nabla g(\mathbf{x})=g(\mathbf{x}), \forall \mathbf{x} \in \mathbb{R}_{++}^{s}$. Once the internal variables $\left(y_{\alpha}, \mathbf{x}_{\alpha}\right)_{\alpha=1}^{p}$, solution of (3.3), are calculated, the fugacity is given by

$$
p_{i}^{\text {equil }}(\mathbf{b})=\exp \left(\nabla g\left(\mathbf{x}_{\alpha}\right)+\ln p_{\text {vapor }, i}\right), \quad i=1, \ldots, s,
$$

where $p_{\mathrm{vapor}, i}$ is the given vapor pressure for pure component $i$ (and depends on the temperature and atmospheric pressure).

Numerical methods for the solution of (3.1) (3.2) have been proposed in [9, 20, 32]. In the remainder of this article, we focus more precisely on this global optimization problem (3.3) and propose two solution methods for the computation of the thermodynamic equilibrium inside each organic particle.

\section{Global Optimization and Computation of Convex Envelopes}

We consider here the optimization problem (3.3) for fixed b. Note that, if $\left\{y_{\alpha}, \mathbf{x}_{\alpha}\right\}_{\alpha=1}^{p}$ is the solution of the minimization problem for $\mathbf{b}$, then for any $c>0,\left\{c y_{\alpha}, \mathbf{x}_{\alpha}\right\}_{\alpha=1}^{p}$ is the solution of the minimization problem for the point $c \mathbf{b}$. Following [4], and without loss of generality, we therefore assume that $\mathbf{e}_{s}^{T} \mathbf{b}=1$ in this section.

We now show that (3.3) is related to the computation of the value of the convex envelope of some function. Let $\Delta_{s}^{\prime}$ be defined by $\Delta_{s}^{\prime}=\left\{\mathbf{x} \in \mathbb{R}^{s}: \mathbf{e}_{s}^{T} \mathbf{x}=1, \mathbf{x} \geq 0\right\}$ and, for $r=s-1$, $\Delta_{r}=\left\{\mathbf{z} \in \mathbb{R}^{r}: \mathbf{e}_{r}^{T} \mathbf{z} \leq 1, \mathbf{z} \geq 0\right\}$. The unit simplex $\Delta_{r}$ can be identified with $\Delta_{s}^{\prime}$ via the mapping $\Pi: \Delta_{r} \rightarrow \Delta_{s}^{\prime}$ such that $\mathbf{z} \rightarrow \mathbf{x}=\mathbf{E}_{s}+Z_{e} \mathbf{z}$, where $\mathbf{E}_{s}=(0, \ldots, 0,1)^{T}$ is the canonical basis vector and $Z_{e}^{T}=\left(\mathbf{I}_{r},-\mathbf{e}_{r}\right)$ with $\mathbf{I}_{r}$ the $r \times r$ identity matrix. We define $\tilde{g}=g \circ \Pi$. The energy $\tilde{g}$ is a non-convex function that is continuously defined on $\Delta_{r}$. In the atmospheric systems under consideration, $\tilde{g}$ is actually convex in the neighborhood of the vertices of $\Delta_{r}$, since the vertices of $\Delta_{r}$ correspond to stable, pure chemical solutions.

Let $P$ be the projection from $\mathbb{R}^{s}$ to $\mathbb{R}^{r}$ defined by $P\left(x_{1}, \ldots, x_{r}, x_{s}\right)=\left(x_{1}, \ldots, x_{r}\right)$, and denote $\mathbf{z}_{\alpha}=P \mathbf{x}_{\alpha}$ for $\alpha=1, \ldots, p$, and $\mathbf{d}=P \mathbf{b}$. The minimization problem (3.3) is equivalent after projection to

$$
\begin{array}{ll}
\min _{y_{\alpha}, \mathbf{z}_{\alpha}} & \sum_{\alpha=1}^{p} y_{\alpha} \tilde{g}\left(\mathbf{z}_{\alpha}\right), \\
\text { s.t. } & \sum_{\alpha=1}^{p} y_{\alpha} \mathbf{z}_{\alpha}=\mathbf{d}, \quad \sum_{\alpha=1}^{p} y_{\alpha}=1, \quad y_{\alpha} \geq 0, \quad \alpha=1, \ldots, p .
\end{array}
$$

Problem (4.1) consists in finding the value of convex envelope of $\tilde{g}$ at the point $\mathbf{d} \in \Delta_{r}$, and the 
value of the optimum is denoted by $(\operatorname{conv} \tilde{g})(\mathbf{d})$. The Carathéodory's theorem (see, e.g., [27]) allows to conclude that the number of phases $p$ is bounded by $s$ :

Theorem 1 (Carathéodory's theorem). For a set $C \neq \emptyset$ in $\mathbb{R}^{r}$, every point of $\operatorname{conv}(C)$ belongs to some simplex with vertices in $C$ and thus can be expressed as a convex combination of $r+1=s$ points of $C$ (not necessarily different). When $C$ is connected, $r$ points suffice. Therefore, for $f: \mathbb{R}^{r} \mapsto \mathbb{R}$,

$$
(\operatorname{conv} f)(\mathbf{d})=\inf \left\{\sum_{\alpha=1}^{r+1} y_{\alpha} f\left(\mathbf{z}_{\alpha}\right) \mid \sum_{\alpha=1}^{r+1} y_{\alpha} \mathbf{z}_{\alpha}=\mathbf{d}, y_{\alpha} \geq 0, \sum_{\alpha=1}^{r+1} y_{\alpha}=1\right\} .
$$

The point $\left(y_{\alpha}, \mathbf{z}_{\alpha}\right)_{\alpha=1, \ldots, p} \in \mathbb{R}^{s p}$ is called a phase splitting of $\mathbf{d}$. A phase splitting is called stable if $y_{\alpha}>0$ for all $\alpha=1, \ldots, p$ and if all $\mathbf{z}_{\alpha}$ are distincts. Thus let us define the sets of indices

$$
\mathcal{I}=\left\{\alpha \in\{1, \ldots, p\}: y_{\alpha}>0\right\} \quad \mathcal{A}=\left\{\alpha \in\{1, \ldots, p\}: y_{\alpha}=0\right\},
$$

as the sets of indices of the inactive and active inequality constraints respectively. Define $p^{\mathcal{I}}=$ card $\mathcal{I}$. A first consequence is that $\left(y_{\alpha}, \mathbf{z}_{\alpha}\right)_{\alpha \in \mathcal{I}}$ is a stable phase splitting of $\mathbf{d}$ if $\left(y_{\alpha}, \mathbf{z}_{\alpha}\right)_{\alpha=1, \ldots, p}$ is a phase splitting of $\mathbf{d}$. The phase diagram $\Delta_{r}$ can be separated into different areas according to the size $p^{\mathcal{I}}$ of the stable phase simplexes, as illustrated later in the numerical results.

A second consequence of the Carathéodory's theorem is that there exists a unique phase simplex $\sum(\mathbf{d})=\operatorname{conv}\left(\mathbf{z}_{1}, \ldots, \mathbf{z}_{p^{\mathcal{I}}}\right)$ with $p^{\mathcal{I}} \leq s$ such that $(\operatorname{conv} \tilde{g})(\mathbf{d})=\sum_{\alpha=1}^{p^{\mathcal{I}}} y_{\alpha} \tilde{g}\left(\mathbf{z}_{\alpha}\right)$ with the barycentric representation $\mathbf{d}=\sum_{\alpha=1}^{p^{\mathcal{I}}} y_{\alpha} \mathbf{z}_{\alpha}, \sum_{\alpha=1}^{p^{\mathcal{I}}} y_{\alpha}=1$ and $y_{\alpha}>0, \forall \alpha \in \mathcal{I}$. Therefore (4.1), and thus (3.3), admit a unique solution, which corresponds to a minimal stable phase simplex.

The Gibbs tangent plane criterion (see, e.g., [22]) states that the affine hyperplane tangent to the graph of $\tilde{g}$ at $\left(\mathbf{z}_{\alpha}, \tilde{g}\left(\mathbf{z}_{\alpha}\right)\right), \forall \alpha \in \mathcal{I}$, lies entirely below the graph of $\tilde{g}$. This hyperplane is called the supporting tangent plane. For the case $s=2, \Delta_{1}=(0,1)$ and a generic representation of $\tilde{g}$ is given in Figure 1. For the points $\mathbf{d}$ located on the left or right part of the graph (see Figure 1 - left and right), $(\operatorname{conv} \tilde{g})(\mathbf{d})=\tilde{g}(\mathbf{d})$, and the stable phase splitting of $\mathbf{d}$ is given by $\left(\mathbf{z}_{1}\right)=(\mathbf{d})$ or by $\left(\mathbf{z}_{2}\right)=(\mathbf{d})$ for the left and right figures respectively. In Figure 1 (middle), the convex envelope of $\tilde{g}$ at $\mathbf{d}$ is no longer superposed with $\tilde{g}$ but follows the segment $\left[\tilde{g}\left(\mathbf{z}_{1}\right), \tilde{g}\left(\mathbf{z}_{2}\right)\right]$, and (conv) $\tilde{g}(\mathbf{d})=y_{1} \tilde{g}\left(\mathbf{z}_{1}\right)+y_{2} \tilde{g}\left(\mathbf{z}_{2}\right)$; the stable phase splitting of $\mathbf{d}$ is $\left(\mathbf{z}_{1}, \mathbf{z}_{2}\right)$ with $y_{1}+y_{2}=1$. In all cases, the affine hyperplane tangent to the graph of $\tilde{g}$ lies below the graph of $\tilde{g}$.

\section{Primal-Dual Interior-Point Method}

Interior-point methods are powerful algorithms for large-scale nonlinear programming (see, e.g., $[5,17])$. The main principle consists in relaxing each inequality constraint $y_{\alpha} \geq 0$ in (3.3), and incorporating $y_{\alpha}$ into a logarithmic barrier term in the objective function. Let us detail this procedure in the particular case of (3.3); note that, even if we are working with $g$ and the variables $\mathbf{x}_{\alpha}$ and $\mathbf{b}$, the figures in this article always illustrate $\tilde{g}$ and the projected variables $\mathbf{z}_{\alpha}$ and $\mathbf{d}$ for convenience. The first order optimality conditions relative to (3.3) read as follows: 

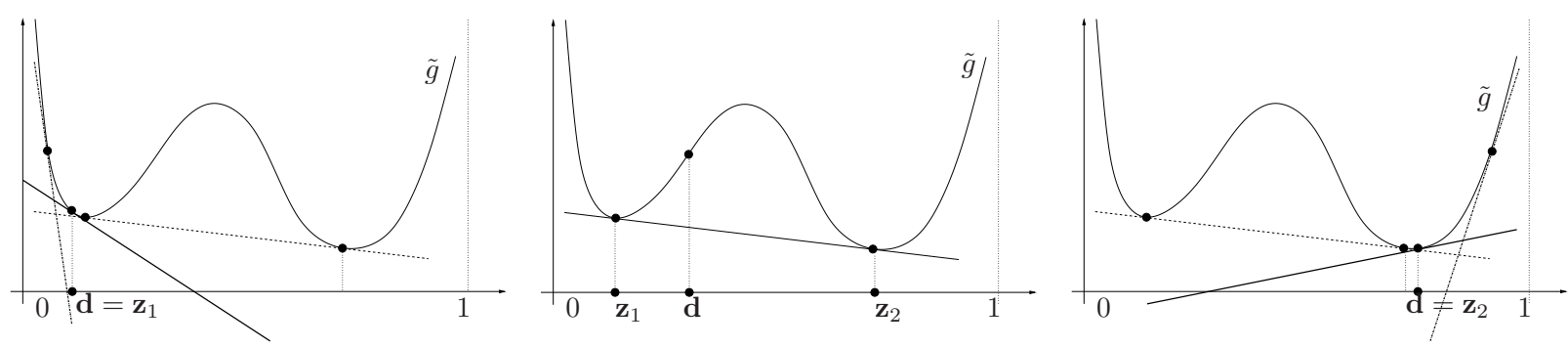

Figure 1: Geometric representation of the computation of the convex envelope and the supporting tangent planes. Left and right: for $\mathbf{d}$ located near the extremities of $[0,1],(\operatorname{conv} \tilde{g})(\mathbf{d})=\tilde{g}(\mathbf{d})$. Middle: for $\mathbf{d}$ located in the middle of $[0,1],(\operatorname{conv} \tilde{g})(\mathbf{d})=y_{1} \tilde{g}\left(\mathbf{z}_{1}\right)+y_{2} \tilde{g}\left(\mathbf{z}_{2}\right)<\tilde{g}(\mathbf{d})$ (with $\left.y_{1}+y_{2}=1\right)$.

$$
\begin{array}{ll}
y_{\alpha}\left(\nabla g\left(\mathbf{x}_{\alpha}\right)+\boldsymbol{\lambda}\right)+\zeta_{\alpha} \mathbf{e}_{s}=\mathbf{0}, & \alpha=1, \ldots, p, \\
g\left(\mathbf{x}_{\alpha}\right)+\boldsymbol{\lambda}^{T} \mathbf{x}_{\alpha}-\theta_{\alpha}=0, & \alpha=1, \ldots, p, \\
\sum_{\alpha=1}^{p} y_{\alpha} \mathbf{x}_{\alpha}=\mathbf{b}, & \alpha=1, \ldots, p, \\
\mathbf{e}_{s}^{T} \mathbf{x}_{\alpha}=1, \quad \mathbf{x}_{\alpha}>0, & \\
y_{\alpha} \theta_{\alpha}=0, \quad y_{\alpha} \geq 0, \quad \theta_{\alpha} \geq 0, & \alpha=1, \ldots, p,
\end{array}
$$

where $\boldsymbol{\lambda} \in \mathbb{R}^{s}$ and $\zeta_{\alpha}, \theta_{\alpha} \in \mathbb{R}, \alpha=1, \ldots, p$, are dual multipliers related to each of the constraints.

Let $\nu>0$ be a given parameter. A primal-dual interior-point method consists in relaxing the last relation of (5.1), so that it becomes

$$
y_{\alpha} \theta_{\alpha}=\nu, \quad y_{\alpha}>0, \quad \theta_{\alpha}>0, \quad \alpha=1, \ldots, p .
$$

The solution of (5.1) (5.2) converges to the solution of the original system (5.1) as $\nu \rightarrow 0$ (see, e.g., [13]). Starting with an initial interior-point parameter $\nu^{0},(5.1)(5.2)$ is solved by applying one Newton iteration, then decreasing the parameter, and repeating the process until convergence is reached $[4,13]$. Let us ignore for now that $y_{\alpha}$ and $\theta_{\alpha}$ must be positive, and let us eliminate the variables $\theta_{\alpha}$. Starting from a well-chosen initial guess $[4,6]$, the Newton method applied to (5.1) (5.2) consists in computing a displacement in $\left(\mathbf{x}_{\alpha}, y_{\alpha}, \lambda, \zeta_{\alpha}\right)$ by solving at each iteration the following linear system (defined by blocks): 


$$
\left(\begin{array}{cccc}
y_{\alpha} \nabla^{2} g\left(\mathbf{x}_{\alpha}\right) & \nabla g\left(\mathbf{x}_{\alpha}\right)+\boldsymbol{\lambda} & y_{\alpha} & \mathbf{e}_{s} \\
\left(\nabla g\left(\mathbf{x}_{\alpha}\right)+\boldsymbol{\lambda}\right)^{T} & \frac{\nu}{y_{\alpha}^{2}} & \mathbf{x}_{\alpha} & \mathbf{0} \\
y_{\alpha}^{T} & \mathbf{x}_{\alpha}^{T} & \mathbf{0} & \mathbf{0} \\
\mathbf{e}_{s}^{T} & \mathbf{0} & \mathbf{0} & \mathbf{0}
\end{array}\right)\left(\begin{array}{c}
\mathbf{p}_{\mathbf{x}_{\alpha}} \\
\mathbf{p}_{y_{\alpha}} \\
\mathbf{p}_{\boldsymbol{\lambda}} \\
\mathbf{p}_{\zeta_{\alpha}}
\end{array}\right)=-\left(\begin{array}{c}
y_{\alpha}\left(\nabla g\left(\mathbf{x}_{\alpha}\right)+\boldsymbol{\lambda}\right)+\zeta_{\alpha} \mathbf{e}_{s} \\
g\left(\mathbf{x}_{\alpha}\right)+\boldsymbol{\lambda}^{T} \mathbf{x}_{\alpha}-\nu / y_{\alpha} \\
\sum_{\alpha=1}^{p} y_{\alpha} \mathbf{x}_{\alpha}-\mathbf{b}, \\
\mathbf{e}_{s}^{T} \mathbf{x}_{\alpha}-1
\end{array}\right)
$$

Finding a solution that satisfies the Karush-Kuhn-Tucker (KKT) system (5.1) (5.2) is actually a difficult problem. Iterative methods for the solution of the linear systems should be avoided as exact solutions are needed in order to avoid the introduction of additional uncertainties. The Gibbs-Duhem relation [28] implies that the blocks $y_{\alpha} \nabla^{2} g\left(\mathbf{x}_{\alpha}\right)$ in (5.3) are not directly invertible, preventing the use of a direct Schur complement method. A projection on the null-space of the constraints is therefore first required, followed by decomposition methods. A new estimate of the solution of (5.1) (5.2) is then proceeded by setting $\mathbf{x}_{\alpha}^{+}=\mathbf{x}_{\alpha}+\tau \mathbf{p}_{\mathbf{x}_{\alpha}}, y_{\alpha}^{+}=y_{\alpha}+\tau \mathbf{p}_{y_{\alpha}}$, $\boldsymbol{\lambda}^{+}=\boldsymbol{\lambda}+\tau \mathbf{p}_{\boldsymbol{\lambda}}, \zeta_{\alpha}^{+}=\zeta_{\alpha}+\tau \mathbf{p}_{\zeta_{\alpha}}$, where $\tau$ is a step-size chosen to ensure that the new primal and dual iterates $y_{\alpha}^{+}$and $\theta_{\alpha}^{+}$remain feasible (positive).

The above procedure is not directly applicable for computation; the difficulty is mainly caused by the combinatorial aspect of the KKT system (5.1), hidden in the complementary slackness conditions: $\theta_{\alpha} \geq 0, y_{\alpha} \geq 0$ and $\theta_{\alpha} y_{\alpha}=0$.

One could attempt to guess the optimal set of inactive constraints $\mathcal{I}=\left\{\alpha=1, \ldots, p: y_{\alpha}^{+}>\right.$ 0 \}, i.e., the set of constraints that are actually inactive at equilibrium. Based on this guess, one could transform (5.1) into a system of nonlinear equations, which is much more computationally tractable. Unfortunately, the set of all possible sets $\mathcal{I}$ grows exponentially with the number of phases $p$ considered.

The interior-point method is therefore coupled with an active sets method for the accurate identification of the set $\mathcal{I}$. Starting with the initial guess $\mathcal{I}^{0}=\{1, \ldots, p\}$, this set is updated at each interior-point iteration. First (5.1) (5.2) is solved for all indices $\alpha \in \mathcal{I}^{k}$ and the variables $\left(y_{\alpha}, \mathbf{x}_{\alpha}\right)$ for $\alpha \notin \mathcal{I}^{k}$ are 'frozen'. Then, if there exists $\alpha \in \mathcal{I}^{k}$ such that $0<y_{\alpha}^{+}<\varepsilon_{y}$ (where $\varepsilon_{y}$ is a given threshold), then the index $\alpha$ is removed from $\mathcal{I}^{k}$ and the corresponding equations are removed from (5.1) (5.2). Reciprocally, if there exists $\alpha \notin \mathcal{I}^{k}$ such that $\theta_{\alpha}^{+}=g\left(\mathbf{x}_{\alpha}^{+}\right)+\left(\mathbf{x}_{\alpha}^{+}\right)^{T} \boldsymbol{\lambda}^{+}<0$, the index $\alpha$ is reintroduced into $\mathcal{I}^{k+1}$ for the next iteration.

Remark 2. At each iteration of the interior-point method, the parameter $\nu^{k}$ is decreased to ultimately reach convergence to the solution of the constrained problem. The rule for decreasing $\nu^{k}$ at each iteration of the interior-point method follows the discussion in [4]. According to numerical experiments, $\nu^{k+1}=0.7 \nu^{k}$ allows good convergence properties.

The complete algorithm for the computation of the convex envelope at one given point $\mathbf{b}$ can be summarized as follows. This algorithm is repeated for each point of some grid covering $\Delta_{r}$, in order to compute a discrete approximation of the convex envelope.

Step 1. Initialization of $\left(y_{\alpha}^{0}, \mathbf{x}_{\alpha}^{0}, \boldsymbol{\lambda}^{0}, \zeta_{\alpha}^{0}\right)$ and $\nu=\nu^{0}$. Set $\mathcal{I}^{0}=\{1, \ldots, p\}$. Then, for $k \geq 0$, execute Steps 2-6 until some stopping criterion is satisfied: 
Step 2. Compute the reduced Newton direction $\left(\mathbf{p}_{\mathbf{x}_{\alpha}}, \mathbf{p}_{y_{\alpha}}, \mathbf{p}_{\boldsymbol{\lambda}}, \mathbf{p}_{\zeta_{\alpha}}\right)$ by solving (5.3) associated with the set of inactive constraint $\mathcal{I}^{k}$ (i.e. by replacing $\alpha=1, \ldots, p$ by $\alpha \in \mathcal{I}^{k}$ ).

Step 3. Compute a step-length $\tau$ to ensure that $y_{\alpha}^{+}>0$.

Step 4. Compute the new iterates $\mathbf{x}_{\alpha}^{k+1}, y_{\alpha}^{k+1}, \boldsymbol{\lambda}^{k+1}, \zeta_{\alpha}^{k+1}$.

Step 5. Update the set $\mathcal{I}^{k} \rightarrow \mathcal{I}^{k+1}$.

Step 6. Compute the new parameter $\nu^{k+1}=0.7 \nu^{k}$.

Numerical results are given in Section 7., for various chemical systems, following [4, 9, 19]. In the next section, we first present an alternative approach based on variational arguments, for the computation of the convex envelopes.

\section{Variational Approach: Applications to Systems with Two Components}

We present here another numerical method based on a variational approach. We tackle only the one-dimensional case in this article, namely the computation of the thermodynamic equilibrium of a chemical system involving two organic chemical components. The numerical algorithm we present here is inspired from [14], and has already been used in other situations, such as nonsmooth eigenvalue problems [8] or non-smooth diffusion operators [7].

\subsection{The Convex Envelope as a Variational Problem}

Let us consider the unit simplex in one dimension, $\Omega=\Delta_{1}=(0,1)$. Let $g \in C^{0}(\bar{\Omega}) \cap C^{\infty}(\Omega)$ ( $g$ non convex), be a given Gibbs free energy function as in the previous sections. The goal is to compute the convex envelope of $g$.

The convex envelope function being the largest convex function that bounds $g$ from below, let us define the functional spaces:

$$
\begin{aligned}
& V_{g}=\left\{v \in H^{2}(\Omega): v(0)=g(0), v(1)=g(1)\right\}, \\
& K_{g}=\left\{v \in V_{g}: v(x) \leq g(x), \text { a.e. } x \in \Omega\right\}, \quad K=\left\{v \in K_{g}: v^{\prime \prime}(x) \geq 0\right\},
\end{aligned}
$$

In particular, the space $V_{0}$ addresses the case of functions with homogeneous Dirichlet boundary conditions. The variational problem we consider here reads as follows: find $u \in K$ solution of

$$
\inf _{v \in K} \frac{1}{2}\left\|v^{\prime \prime}\right\|_{L^{2}(\Omega)}^{2}
$$

The solution to this problem is bounded from above by $g$ and minimizes the curvature in the $L^{2}$-sense, by minimizing the second derivative. It satisfies appropriate boundary conditions, and 
is convex; it gives therefore the convex envelope. In the sequel, we propose an augmented Lagrangian based algorithm for the solution of (6.2) that decouples the differential operators from the constraints introduced in the convex set $K$.

\subsection{Augmented Lagrangian Algorithm}

The numerical solution of (6.2) is addressed following the approach initially presented for instance in $[14,15]$. Let us define first $q=v^{\prime \prime} \in L^{2}(\Omega)$. Problem (6.2) is equivalent to

$$
\min _{(v, q) \in \mathcal{K}} \frac{1}{2}\left\|v^{\prime \prime}\right\|_{L^{2}(\Omega)}^{2}
$$

where $\mathcal{K}=\left\{(v, q) \in K_{g} \times L^{2}(\Omega): v^{\prime \prime}-q=0, q \geq 0\right\}$. Based on (6.3), we look for a saddle point of the augmented Lagrangian functional defined by

$$
\mathcal{L}_{\gamma}(v, q, \mu)=\frac{1}{2}\left\|v^{\prime \prime}\right\|_{L^{2}(\Omega)}^{2}+\frac{\gamma}{2}\left\|v^{\prime \prime}-q\right\|_{L^{2}(\Omega)}^{2}+\int_{0}^{1} \mu\left(v^{\prime \prime}-q\right) d x
$$

where $\gamma \geq 0$ is a positive parameter. Define $L_{+}^{2}(\Omega)=\left\{q \in L^{2}(\Omega), q \geq 0\right\}$. Namely, we are looking for $\{u, p, \lambda\} \in K_{g} \times L_{+}^{2}(\Omega) \times L^{2}(\Omega)$ such that

$$
\mathcal{L}_{\gamma}(u, p, \mu) \leq \mathcal{L}_{\gamma}(u, p, \lambda) \leq \mathcal{L}_{\gamma}(v, q, \lambda),
$$

for all $\{v, q, \mu\} \in K_{g} \times L_{+}^{2}(\Omega) \times L^{2}(\Omega)$. An iterative Uzawa algorithm, that is reminiscent of the algorithm $A L G 2$ discussed in $[14,16]$, is advocated. Let $u^{-1} \in V_{g}$ and $\lambda^{0} \in L^{2}(\Omega)$ be given arbitrary functions (typically $\lambda^{0}=0$ ). Then, for $k \geq 0$.

(a) Solve

$$
p^{k}=\arg \min _{q \in L_{+}^{2}(\Omega)}\left[\frac{\gamma}{2} \int_{\Omega}|q|^{2} d x-\int_{\Omega} X^{k} q d x\right],
$$

where $X^{k}=\gamma\left(u^{k-1}\right)^{\prime \prime}+\lambda^{k} \in L^{2}(\Omega)$. This problem admits a unique minimizer, with a closedform solution, as $(6.5)$ can be solved point-wise a.e. $x$ in $\Omega$ (see e.g. [11, 12]). Its solution reads:

$$
p^{k}(x)=\left(\frac{X^{k}(x)}{\gamma}\right)^{+}, \quad \text { a.e. } x \in \Omega
$$

where $(\psi)^{+}:=\max (\psi, 0)$.

(b) Solve

$$
u^{k}=\arg \min _{v \in K_{g}}\left[\frac{\gamma+1}{2} \int_{\Omega}\left|v^{\prime \prime}\right|^{2} d x-\int_{\Omega} Y^{k} v^{\prime \prime} d x\right]
$$


where $Y^{k}=\gamma p^{k}-\lambda^{k} \in L^{2}(\Omega)$. The first order optimality conditions corresponding to (6.7) read as follows: find $u^{k} \in K_{g}$ such that

$$
(\gamma+1) \int_{\Omega}\left(u^{k}\right)^{\prime \prime} v^{\prime \prime} d x=\int_{\Omega} Y^{k} v^{\prime \prime} d x
$$

If we neglect the constraint in the definition of $K_{g}$, the strong formulation corresponding to (6.8) is the biharmonic problem:

$$
\left\{\begin{array}{l}
(\gamma+1)\left(u^{k}\right)^{(4)}(x)=\left(Y^{k}\right)^{\prime \prime}(x), \\
u^{k}(0)=u^{k}(1)=\left(u^{k}\right)^{\prime \prime}(0)=\left(u^{k}\right)^{\prime \prime}(1)=0 .
\end{array} \quad x \in \Omega,\right.
$$

Numerical techniques for the solution of (6.8) are discussed in Sections 6.3. and 6.4., by using finite elements techniques.

(c) Update the multipliers $\lambda^{k} \in L^{2}(\Omega)$ :

$$
\lambda^{k+1}=\lambda^{k}+\gamma\left(\left(u^{k}\right)^{\prime \prime}-p^{k}\right)
$$

until convergence is reached. Typically the stopping criterion is $\left\|u^{k}-u^{k-1}\right\|_{L^{2}(\Omega)}<\varepsilon$, where $\varepsilon$ is a given tolerance.

\subsection{Finite Element Discretization}

Finite elements techniques are used for the numerical implementation of algorithm (6.5)-(6.9). Let $N>0$ be a given integer and $h=(N+1)^{-1}$ be a discretization step. The discretization of the domain $\Omega=(0,1)$ is given by $\mathcal{T}_{h}=\left\{x_{0}, x_{1}, \ldots, x_{N}, x_{N+1}\right\}$, with $x_{i}=i h, i=0, \ldots, N+1$ $\left(x_{0}=0, x_{N+1}=1\right)$. The finite element spaces are defined by

$$
\begin{aligned}
& V_{h}=\left\{v \in C^{0}(\bar{\Omega}):\left.v\right|_{\left[x_{i}, x_{i+1}\right]} \in \mathbb{P}_{1}, \forall i=0, \ldots, N\right\}, \\
& V_{g h}=\left\{v \in V_{h}, v(0)=g_{h}(0), v(1)=g_{h}(1)\right\}, \\
& K_{g h}=\left\{v \in V_{g h}: v\left(x_{i}\right) \leq g_{h}\left(x_{i}\right), i=0, \ldots, N+1\right\}, \\
& K_{h}=\left\{v \in K_{g h}: D_{h}^{2} v\left(x_{i}\right) \geq 0, i=0, \ldots, N+1\right\},
\end{aligned}
$$

where $D_{h}^{2} v \in V_{h}$ is defined by the nodal values:

$$
D_{h}^{2} v\left(x_{i}\right)=\frac{v\left(x_{i+1}\right)-2 v\left(x_{i}\right)+v\left(x_{i-1}\right)}{h^{2}} \simeq u^{\prime \prime}\left(x_{i}\right),
$$

with appropriate corrections at the end points of the interval $(0,1)$, and $g_{h}$ is the piecewise affine interpolant of $g$. In particular, the space $V_{0 h}$ covers the case $g_{h}=0$. Actually $D_{h}^{2} v$ is a second order approximation of $u^{\prime \prime}$ when the grid points are equally spaced. Let $\varphi_{j}, j=1, \ldots, N$ be the finite element basis functions of $V_{0 h}$, based on the discretization $\mathcal{T}_{h}$. For $v_{h}, w_{h} \in V_{h}$, the integral $\int_{\Omega} v_{h} w_{h} d x$ 
is approximated by the trapezoidal rule and we define $\left(v_{h}, w_{h}\right)_{0 h}=\frac{1}{3} \sum_{j=1}^{N_{n}} A_{j} v_{h}\left(P_{j}\right) w_{h}\left(P_{j}\right)$, where $P_{j}$ is a vertex of $\mathcal{T}_{h}$ and $A_{j}$ is the length of the segment which is the union of those segments $\left[x_{i}, x_{i+1}\right]$ which have $P_{j}$ as a common vertex. We denote the corresponding norm by $\left\|v_{h}\right\|_{0 h}=\left(v_{h}, v_{h}\right)_{0 h}^{1 / 2}$.

We look for a saddle point of the discrete augmented Lagrangian functional defined by

$$
\mathcal{L}_{\gamma h}\left(v_{h}, q_{h}, \mu_{h}\right)=\frac{1}{2}\left\|D_{h}^{2} v_{h}\right\|_{0 h}^{2}+\frac{\gamma}{2}\left\|D_{h}^{2} v_{h}-q_{h}\right\|_{0 h}^{2}+\left(\mu_{h}, D_{h}^{2} v_{h}-q_{h}\right)_{0 h},
$$

Define $V_{+h}=\left\{q \in V_{h}, q\left(x_{i}\right) \geq 0, i=0, \ldots, N+1\right\}$. We are looking for $\left\{u_{h}, p_{h}, \lambda_{h}\right\} \in K_{g h} \times$ $V_{+h} \times V_{h}$ such that

$$
\mathcal{L}_{\gamma h}\left(u_{h}, p_{h}, \mu_{h}\right) \leq \mathcal{L}_{\gamma h}\left(u_{h}, p_{h}, \lambda_{h}\right) \leq \mathcal{L}_{\gamma h}\left(v_{h}, q_{h}, \lambda_{h}\right),
$$

for all $\left\{v_{h}, q_{h}, \mu_{h}\right\} \in K_{g h} \times V_{+h} \times V_{h}$. The discrete version of the algorithm (6.5)-(6.9) consists in looking for approximations $u_{h} \in K_{g h}, p_{h} \in V_{+h}$ and $\lambda_{h} \in V_{h}$ of $u, p$ and $\lambda$ respectively that are computed according to the following discretized algorithm. Let $u_{h}^{-1} \in V_{g h}$ and $\lambda_{h}^{0} \in V_{h}$ (typically $\left.\lambda_{h}^{0}=0\right)$. For $k \geq 0$ :

(a) Find $p_{h}^{k} \in V_{+h}$ defined by the nodal values $p_{i}^{k}:=p_{h}^{k}\left(x_{i}\right)$ :

$$
p_{i}^{k}=\left(\frac{X_{i}^{k}}{\gamma}\right)^{+}, \quad i=0, \ldots, N+1
$$

where $X_{i}^{k}=\gamma D_{h}^{2} u_{h}^{k-1}\left(x_{i}\right)+\lambda_{i}^{k}, i=0, \ldots, N+1$.

(b) Solve

$$
u_{h}^{k}=\arg \min _{v \in K_{g h}}\left[\frac{\gamma+1}{2}\left\|D_{h}^{2} v\right\|_{0 h}^{2}-\left(Y_{h}^{k}, D_{h}^{2} v\right)_{0 h}\right]
$$

where $Y_{h}^{k}=\gamma p_{h}^{k}-\lambda_{h}^{k} \in V_{h}$. The solution to (6.14) consists in finding first $\tilde{u}_{h}^{k} \in V_{g h}$ such that:

$$
\left(D_{h}^{2} \tilde{u}_{h}^{k}, D_{h}^{2} v\right)_{0 h}=\frac{1}{\gamma+1}\left(Y_{h}^{k}, D_{h}^{2} v\right)_{0 h}, \quad \forall v \in V_{0 h},
$$

and then project the solution on the convex set $K_{g h}$ by setting, for $i=1, \ldots, N$ :

$$
u_{h}^{k}\left(x_{i}\right)= \begin{cases}\tilde{u}_{h}^{k}\left(x_{i}\right), & \text { if } \tilde{u}_{h}^{k}\left(x_{i}\right)<g\left(x_{i}\right), \\ g\left(x_{i}\right), & \text { otherwise. }\end{cases}
$$

The solution of the discrete elliptic biharmonic problem (6.15) is detailed in Section 6.4..

(c) Update the multipliers $\lambda_{h}^{k} \in V_{h}$ :

$$
\lambda_{h}^{k+1}=\lambda_{h}^{k}+\gamma\left(D_{h}^{2} u_{h}^{k}-p_{h}^{k}\right)
$$


until convergence is reached. The discrete stopping criterion is $\left\|u_{h}^{k}-u_{h}^{k-1}\right\|_{0 h}<\varepsilon$, where $\varepsilon$ is a given tolerance.

\subsection{Solution of Discrete Biharmonic Problems}

The problem equivalent to $(6.15)$ at the continuous level belongs to the category of generic biharmonic problems that can be written as follows:

$$
u \in V_{g}, \int_{0}^{1} u^{\prime \prime} v^{\prime \prime} d x=\int_{0}^{1} f v^{\prime \prime} d x, \forall v \in V_{0}
$$

(where $f \in H^{2}(\Omega)$ ). In order to solve such a biharmonic problem, we set $w=-u^{\prime \prime}$ and introduce the additional boundary conditions $u^{\prime \prime}(0)=u^{\prime \prime}(1)=0$. We decompose the biharmonic problem and solve two successive Laplacian problems (see, e.g., [11]), namely:

$$
\begin{aligned}
& w \in V_{0}, \quad \int_{0}^{1} w^{\prime} v^{\prime} d x=\int_{0}^{1} f v^{\prime \prime} d x, \quad \forall v \in V_{0}, \\
& u \in V_{g}, \quad \int_{0}^{1} u^{\prime} v^{\prime} d x=\int_{0}^{1} w v d x, \quad \forall v \in V_{0} .
\end{aligned}
$$

The discrete version of (6.17) (6.18) consists in solving successively:

$$
\begin{aligned}
& w_{h} \in V_{0 h}, \quad \int_{\Omega} w_{h}^{\prime} v_{h}^{\prime} d x=\left(f^{\prime \prime}, v_{h}\right)_{0 h}+f(1) v_{h}^{\prime}(1)-f(0) v_{h}^{\prime}(0), \quad \forall v_{h} \in V_{0 h}, \\
& u_{h} \in V_{g h}, \quad \int_{\Omega} u_{h}^{\prime} v_{h}^{\prime} d x=\left(w_{h}, v_{h}\right)_{0 h}, \quad \forall v_{h} \in V_{0 h},
\end{aligned}
$$

where the computation of the right-hand side of (6.19) is done by integration by parts. In our case $f=Y_{h}^{k} /(\gamma+1)$ is differentiated twice at the discrete level, similarly to (6.11).

The solution of (6.15) requires the solution of two Laplacian problems at each iteration of the Uzawa algorithm; (6.19) (6.20) correspond to the two linear systems:

$$
\mathbf{A} \mathbf{w}=\mathbf{b}^{1}, \quad \mathbf{A u}=\mathbf{b}^{2}
$$

where $\mathbf{A}=\left(A_{i j}\right)_{i, j=1}^{N}$ is defined by $A_{i j}=\int_{\Omega} \varphi_{i}^{\prime} \varphi_{j}^{\prime} d x, \mathbf{w}=\left(w_{i}\right)_{i=1}^{N}, \mathbf{u}=\left(u_{i}\right)_{i=1}^{N}$, and the righthand sides $\mathbf{b}^{k}=\left(b_{i}^{k}\right)_{i=1}^{N}$ are defined by:

$$
\begin{aligned}
& b_{j}^{1}=h\left(\frac{f\left(x_{j+1}\right)-f\left(x_{j}\right)+f\left(x_{j-1}\right)}{h^{2}}\right)-\frac{1}{h} f(0) \delta_{j 1}-\frac{1}{h} f(1) \delta_{j N}, \\
& b_{j}^{2}=h w_{i},
\end{aligned}
$$

where $\delta_{j k}$ is the Kronecker symbol. The linear systems (6.21) are solved with direct methods, as the Cholesky decomposition of $\mathbf{A}$ is performed once and for all at the beginning of the algorithm. 
Remark 3. The finite element techniques presented in the previous two sections also hold when the grid points are not equally spaced. Let us denote by $h_{i}=x_{i+1}-x_{i}, i=0,1, \ldots, N$. The computation of the second derivatives (6.11) is replaced by:

$$
D_{h}^{2} v\left(x_{i}\right)=\frac{h_{i-1} v\left(x_{i+1}\right)-\left(h_{i}+h_{i-1}\right) v\left(x_{i}\right)+h_{i} v\left(x_{i-1}\right)}{h_{i} h_{i-1}\left(\frac{h_{i}+h_{i-1}}{2}\right)} \simeq u^{\prime \prime}\left(x_{i}\right) .
$$

Five point finite differences formulas are used to estimate the derivatives at the extremities of the interval $(0,1)$. As a consequence, the second order convergence for the approximation of the second derivatives is lost, which influences the convergence properties of the Uzawa algorithm. The rigidity matrix $\mathbf{A}$ in (6.21) is also modified accordingly.

\section{Numerical Results}

Numerical results are presented for the dimensions $r=1,2$ and 3 (corresponding to a chemical system of $s=2,3$ and 4 components respectively). All computations are executed on an Intel processor of $2.4 \mathrm{GHz}$ and $2 \mathrm{~GB}$ of RAM memory. We consider the following chemical components: pinic acid $\left(\mathrm{C}_{9} \mathrm{H}_{14} \mathrm{O}_{4}\right)$, 1-hexacosanol $\left(\mathrm{C}_{26} \mathrm{H}_{54} \mathrm{O}\right)$, n-propanol $\left(\mathrm{C}_{3} \mathrm{H}_{8} \mathrm{O}\right)$ and water $\left(\mathrm{H}_{2} \mathrm{O}\right)$ at temperature $298.15[\mathrm{~K}]$ and pressure 1 [atm]. Two components systems are constructed by taking pairs of these chemical components. The Gibbs free energy $g$ for organic aerosol particles is modeled by using the UNIFAC model (see [4, 6] and references therein for details). This semi-empirical model consists of parametrized functions which account for short and long term interactions between chemical components. The parameters are calibrated from experimental data (typically in smog chambers). The chemical components considered in this section are benchmark examples of atmospheric species commonly used by both experimentalists and modelers in atmospheric science. Thermodynamic models for the Gibbs free energy have been derived for all these systems.

\subsection{Interior-Point Method}

Figure 2 illustrates the computation of the convex envelope for the water-hexacosanol system. The (normalized) Gibbs free energy function is illustrated by the bold line, while the gray segment illustrates the supporting tangent plane. In Figure 2 and the figures that follow, the abscissa axis corresponds to the fraction of hexacosanol in the system: the system is composed of $100 \%$ of water at the left extremity of $(0,1)$, and of $100 \%$ of hexacosanol ( $0 \%$ of water) at the right extremity. Generally speaking, the vertices of $\Delta_{1}$ correspond to pure solutions, and any point between 0 and 1 corresponds to a mixing of these two components.

Figure 3 illustrates the computation of the convex envelope for the pinic acid-hexacosanol system, by using a dynamic approach detailed in $[9,19,20]$. The supporting tangent planes are illustrated at various points $d$. The union of these cuts determines the convex hull of the Gibbs free 


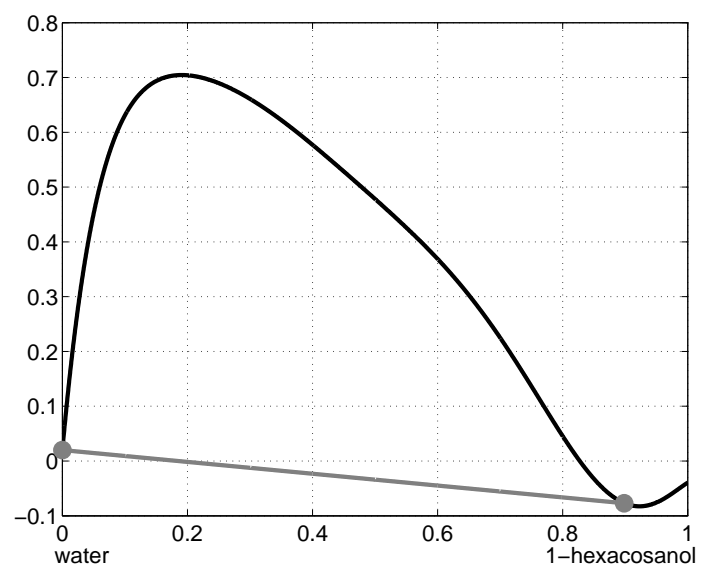

Figure 2: Convex envelope of the Gibbs free energy $\tilde{g}$ for the two-components system including water and 1-hexacosanol: Gibbs free energy (bold line) and supporting tangent plane and contact points (gray line).
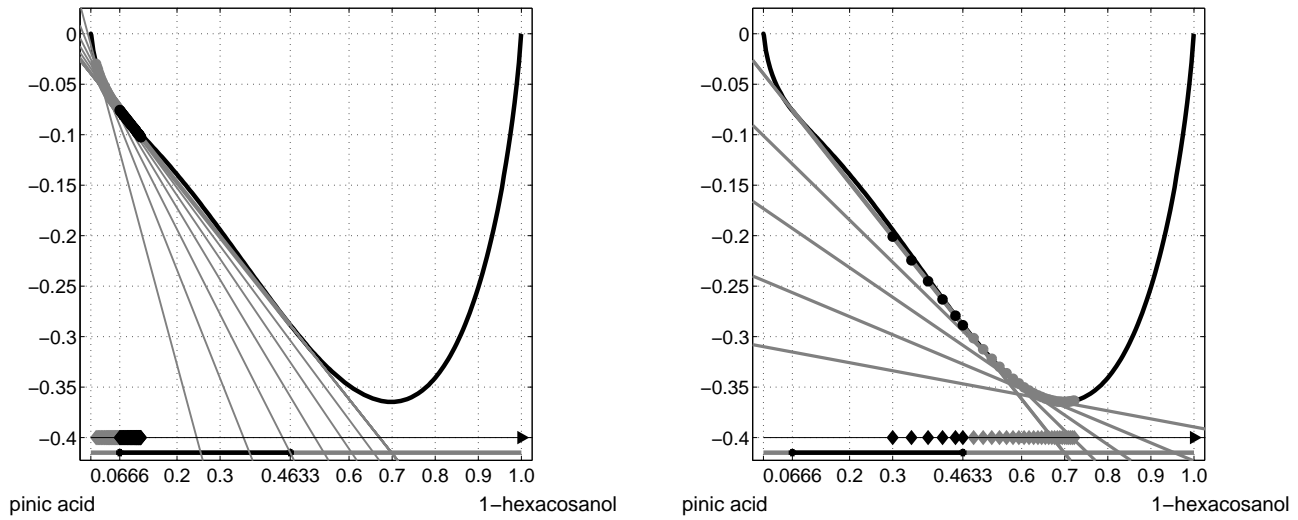

Figure 3: Convex envelope of the Gibbs free energy $\tilde{g}$ for the two-components system including pinic acid and 1-hexacosanol: Gibbs free energy (bold line) and supporting tangent planes for various values of $\mathbf{d}$ (gray lines).

energy function. For such two components systems, the possible size of the stable phase splitting is either one (at both extremities of $\Delta_{1}$ ), or two (in the middle region).

Figure 4 illustrates the convex envelope of the Gibbs free energy of the three components system composed of pinic acid, 1-hexacosanol and water. The vertices of $\Delta_{2}$ correspond to pure solutions of pinic acid, hexacosanol and water respectively. The labels 1,2 and 3 correspond to the size of the stable phase splittings in each particular point of the simplex $\Delta_{2}$. The decomposition of $\Delta_{2}$ into these regions labeled 1,2 or 3 is called a phase diagram. In the region labeled 3 , the coordinates of the variables $\mathbf{z}_{\alpha}$ correspond to the vertices of the inner triangle; in the region labeled 2, the variables $\mathbf{z}_{\alpha}$ are located at the extremity of the supporting segment. Figure 4 show 
two different Gibbs free energy topologies, obtained for two different parametrizations of $g$, for the same chemical system called vapor-liquid equilibrium and liquid-liquid equilibrium systems (see [4] and references therein for more details). This illustrates the sensitivity of the energy function with respect to the experimental modeling and calibration, and justifies the need of a robust computation of the convex envelopes that is suitable to various situations. The computation of such a phase diagram requires to compute the value of the convex envelope on a grid of points of $\Delta_{2}$. The total computational cost is $20 \mathrm{~s}$. when using a grid of $\frac{1}{2}(100 \times 100)$ grid points; each of the computation requires $25-30$ iterations in average (for a tolerance of $10^{-7}$ on the residual).
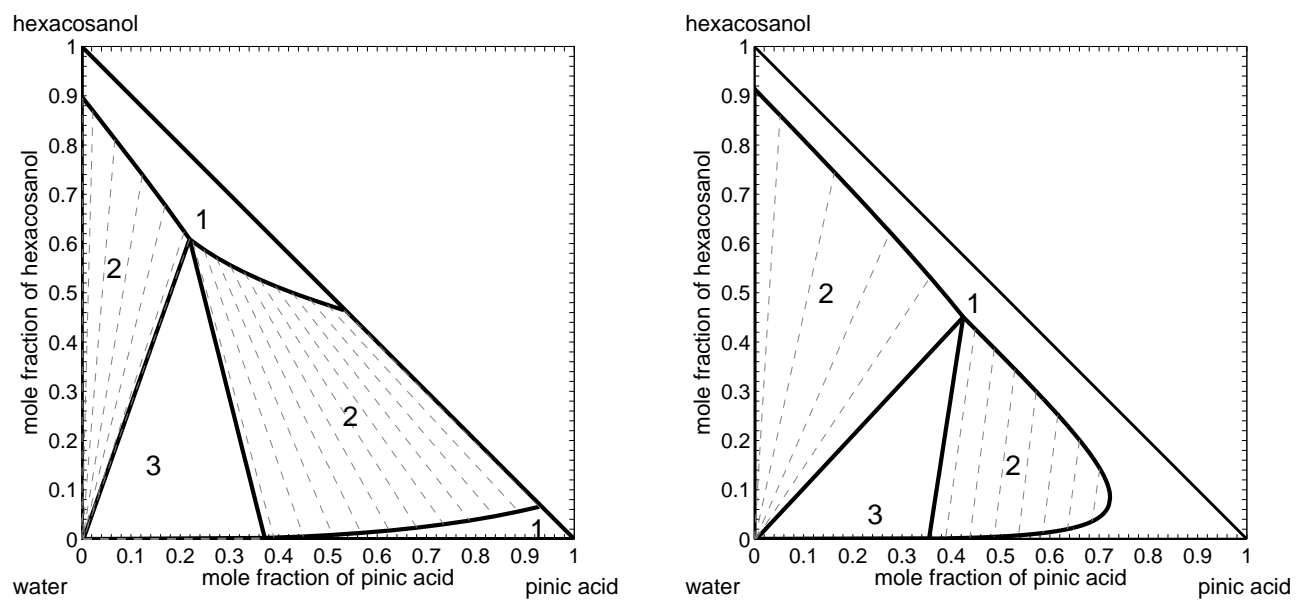

Figure 4: Convex envelopes of the Gibbs energy for three-components system including water, pinic acid and 1-hexacosanol, with two different sets of interaction parameters for the UNIFAC model for $g$.

Finally, Figure 5 is extracted from [19] and visualizes the computation of the convex envelope for a four components chemical system (composed of water, pinic acid, 1-hexacosanol and npropanol) illustrated in $\Delta_{3}$. The value of the convex envelope is computed for a sequence of points located on a trajectory in $\Delta_{3}$ modeled in $[1,9,20]$. The trajectory is projected on the sides of $\Delta_{3}$ for visualization purposes. The changes of color in the trajectory indicate the size changes of the stable phase simplex. The computation of the convex envelope at one point of $\Delta_{3}$ typically takes 40 iterations (for a tolerance of $10^{-7}$ on the residual); the computation cost is $0.0369 \mathrm{~s}$. per grid point in average.

When increasing the number of chemical components, the interior-point method also succeeds in computing the value of the convex envelope at a given point $\mathbf{b}$. For instance, for a system composed of 18 chemical components, the typical cost for each grid point is $0.1799 \mathrm{~s}$. for 41 iterations in average. This illustrates the excellent stability properties of the algorithm, as the number of iterations remains stable, while the computational cost increases accordingly to the size of the linear system. 


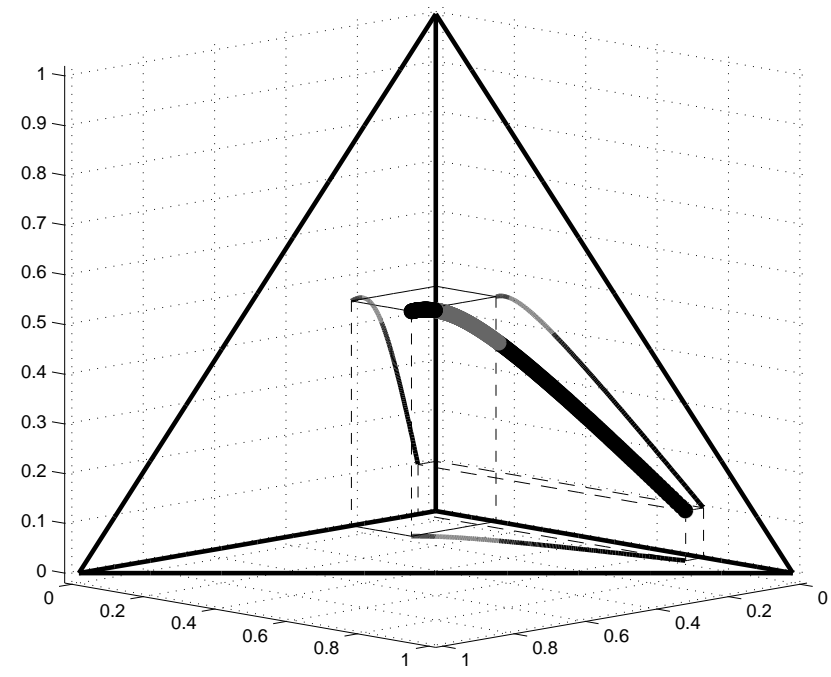

Figure 5: Convex envelope of the Gibbs energy for four-components system including water, pinic acid, 1-hexacosanol and n-propanol (extracted from [19]).

\subsection{Variational Approach}

The augmented Lagrangian algorithm is first benchmarked for simple (polynomial) functions $g$. The corresponding results are visualized in Figure 6 when taking a grid with $N=100$ points equally spaced on $[0,1](h=1 / 101)$. The number of iterations to reach convergence is typically around $10-20$ iterations (with a stopping criterion of $\varepsilon=10^{-8}$ ). Numerical results illustrate that the proposed method does not fail for a large number of configurations.

Real applications to organic aerosol particles are considered again when the function $g$ is generated by atmospheric models (i.e. the UNIFAC model, see [4] in references therein for details). Let us consider two-components systems, composed namely by water and pinic acid, water and 1-hexacosanol, and pinic acid and 1-hexacosanol. The function $g$ is defined on a regular grid of 2002 grid points equally spaced on $[0,1]$. Numerical results in Figure 7 match the ones obtained with the interior-point method and validates the accuracy of the computation of the liquid-liquid equilibrium. The number of iterations to reach convergence is around $20-50$ (with a stopping criterion of $\varepsilon=10^{-8}$ ).

We can overcome the drawback of introducing a large number of grid points, (since only the contact points of the function with the tangent plane are really needed to compute the convex envelope) by using not equally spaced grid points. Figure 8 visualizes results on polynomial functions when the grid points are randomly perturbed around equally spaced positions in $[0,1]$. As outlined in Remark 3, the finite element techniques apply when the grid points are not equally spaced, but the approximation of the second derivatives with centered finite differences is only first order accurate.

Figure 9 illustrates the computation of the convex envelope for the case of water-pinic acid system with grid points that are not equally spaced. The nonuniform finite element discretization 


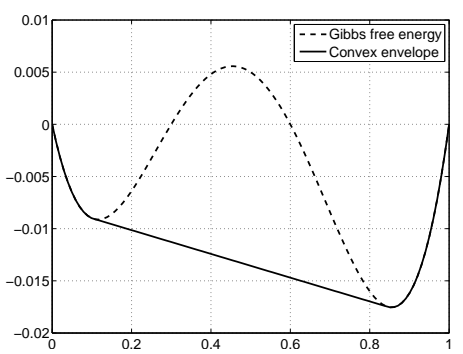

$\tilde{g}(x)=-x(1-x)\left(\frac{3}{10}-x\right)\left(\frac{6}{10}-x\right)$

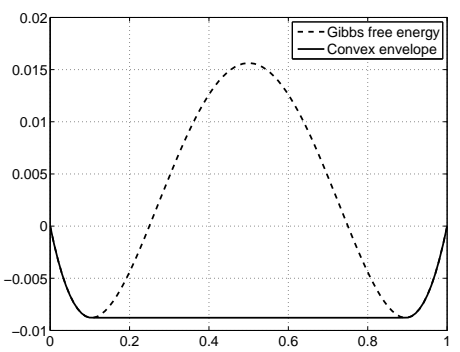

$\tilde{g}(x)=-x(1-x)\left(\frac{1}{4}-x\right)\left(\frac{3}{4}-x\right)$

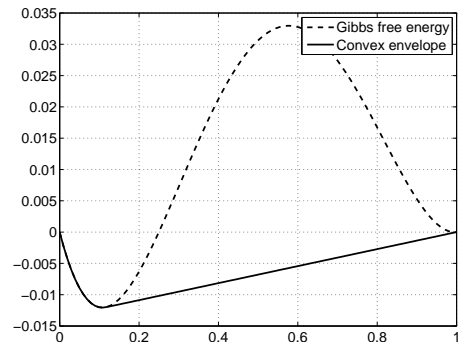

$\tilde{g}(x)=-x(1-x)\left(\frac{1}{4}-x\right)\left(\frac{99}{100}-x\right)$

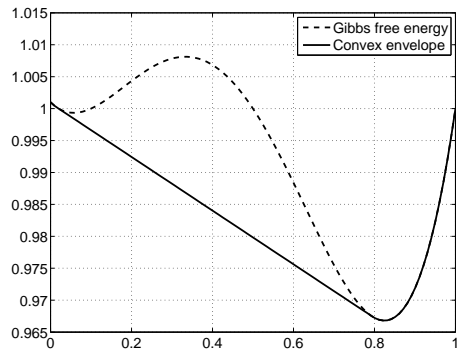

$\tilde{g}(x)=\left(x-\frac{2}{100}\right)\left(x-\frac{1}{10}\right)\left(x-\frac{1}{2}\right)(x-1)+1$

Figure 6: Numerical computation of convex envelopes of several polynomial functions $(N=100)$.

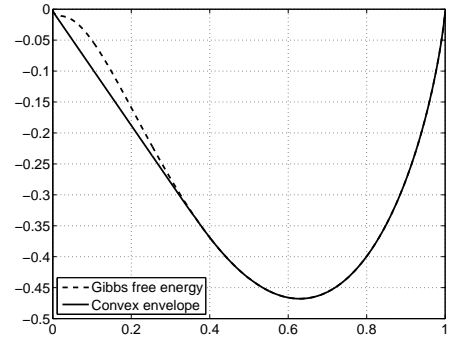

water - pinic acid

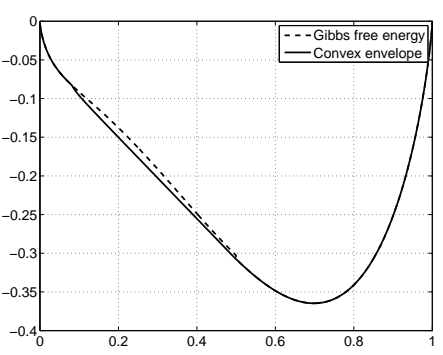

1-hexacosanol - pinic acid

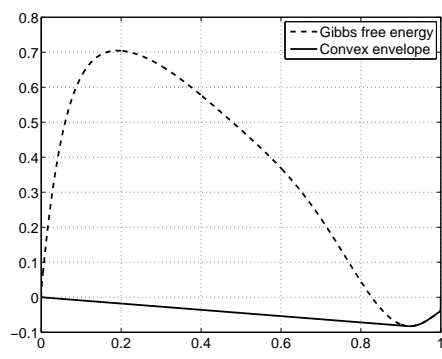

water - 1-hexacosanol

Figure 7: Convex envelopes of the Gibbs energy for two-components systems: Gibbs free energy (dashed line) and convex envelope $(N=2002)$. Left: water and pinic acid; middle: 1 -hexacosanol and pinic acid; right: water and 1-hexacosanol.

is such that a higher density of grid points are located in the convex regions of $g$ (allowing to go from $N=2002$ to $N=148$ grid points, without losing the quality of the solution).

Both the interior-point method and the augmented Lagrangian algorithm have advantages and drawbacks. The interior-point method shows excellent computational performance, and a flexibility that allows to tackle the problem in any dimension. On the other hand, as it only compute the contact point between a function and its convex envelope, it lacks a thorough functional frame- 


$$
\tilde{g}(x)=-x(1-x)\left(\frac{1}{4}-x\right)\left(\frac{3}{4}-x\right)
$$

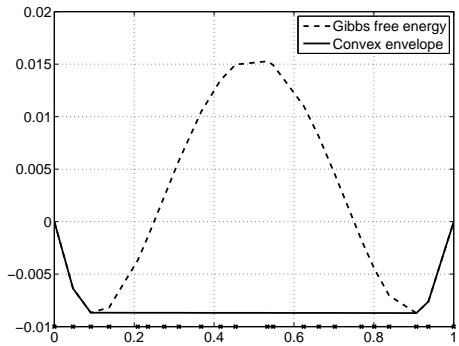

$N=20$

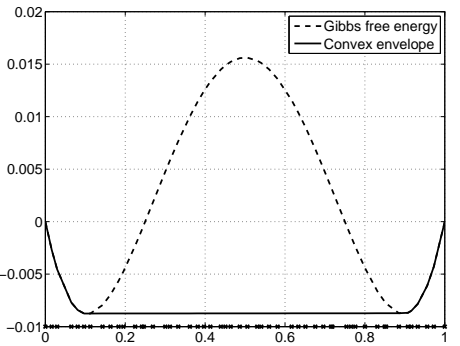

$N=50$

$$
\tilde{g}(x)=\left(x-\frac{2}{100}\right)\left(x-\frac{1}{10}\right)\left(x-\frac{1}{2}\right)(x-1)+1
$$

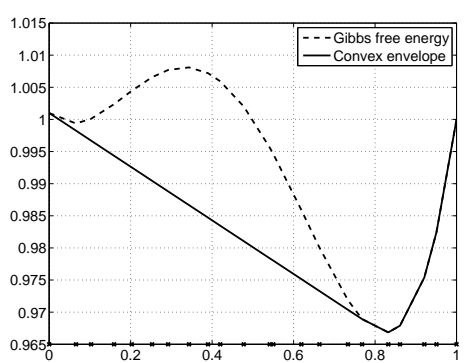

$N=20$

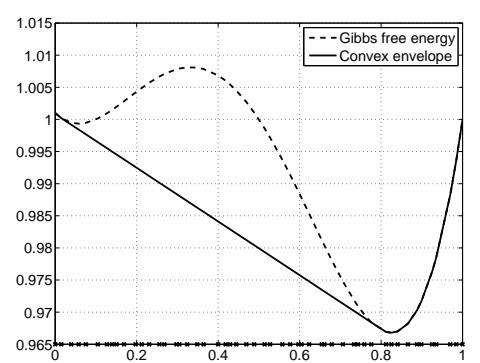

$N=50$

Figure 8: Numerical computation of convex envelopes of polynomial functions for not equally spaced grid points ( $N=20$ and $N=50$ respectively). The density of grid points is represented on an horizontal line below the graph of the function.

work, which forbids us to develop an approximation theory of the problem and error estimates. The variational approach is less general, as it tackle (for now) only two components systems. It introduces a whole variational framework that may allow us to obtain approximation results. Although it is less computationally efficient a priori, adaptive meshing allows this approach to be competitive. One of the achievements of this article has been therefore to link techniques from operations research to a framework from functional analysis.

\section{Conclusion}

A general mathematical model for atmospheric flow has been presented. After generic space discretization and an appropriate operator splitting algorithm, the modeling of aerosol processes leads to the solution of a global optimization problem at each grid point, that corresponds to the com- 


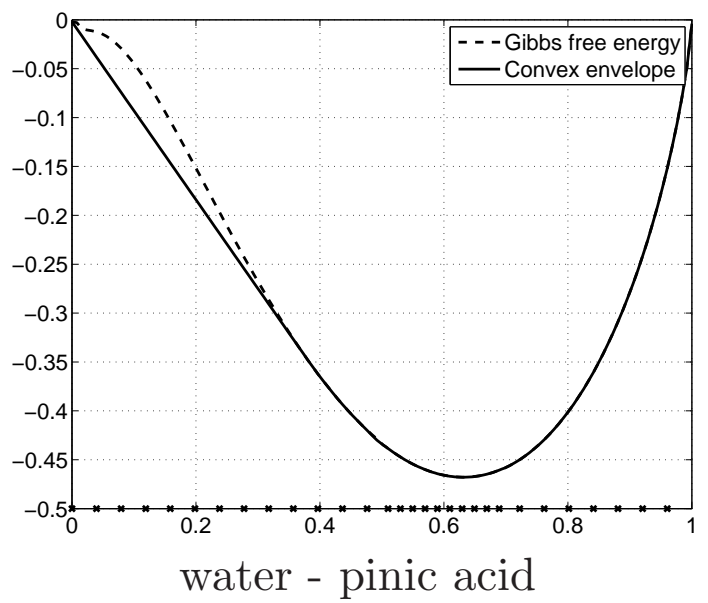

Figure 9: Convex envelopes of the Gibbs energy for two-components systems: Gibbs free energy (dashed line) and convex envelope ( $N=148$, water and pinic acid). The density of grid points is represented on an horizontal line below the graph of the function (one marker every five grid points).

putation of the convex envelope of some energy function when considering organic particles only. Two numerical frameworks have been introduced; the first one relies on an interior-point method, while the second relies on a variational formulation, an appropriate augmented Lagrangian, and an Uzawa-type iterative algorithm that allows to decouple differential operators and nonlinear constraints.

Numerical results in several dimensions have illustrated the efficiency of the interior-point method in any spatial dimensions. On the other hand, the variational method has produced very promising results for two components systems. Extension for multiple components requires the use of numerical techniques for fully or implicitly nonlinear equations, and will be addressed in future works. The augmented Lagrangian approach will be extended in the future for three components systems, and adaptive refinement of the grid will be investigated, together with algorithms for the tracking of convex regions of the energy function.

\section{Acknowledgments}

The author acknowledges the partial support of the National Science Foundation Grant NSF DMS0913982, the US Environmental Protection Agency Grant X-83234201, and MATHICSE at the Ecole Polytechnique Fédérale de Lausanne (part of this work has been achieved during his sabbatical leave in 2009-2010 at EPFL). The author thanks Prof. Roland Glowinski and Prof. Jiwen He (University of Houston), Prof. Lexing Ying (UT Austin) for fruitful discussions, and Dr Chantal Landry (WIAS, Berlin) for her invaluable contribution. 


\section{References}

[1] N. R. Amundson, A. Caboussat, J. W. He, C. Landry, J. H. Seinfeld. A dynamic optimization problem related to organic aerosols. C. R. Acad. Sci., 344 (2007), No. 8, 519-522.

[2] N. R. Amundson, A. Caboussat, J. W. He, C. Landry, C. Tong, J. H. Seinfeld. A new atmospheric aerosol phase equilibrium model (UHAERO): organic systems. Atmos. Chem. Phys., 7 (2007), 4675-4698.

[3] N. R. Amundson, A. Caboussat, J. W. He, A. V. Martynenko, J. H. Seinfeld, K. Y. Yoo. A new inorganic atmospheric aerosol phase equilibrium model (UHAERO). Atmos. Chem. Phys., 6 (2006), 975-992.

[4] N. R. Amundson, A. Caboussat, J. W. He, J. H. Seinfeld. Primal-dual interior-point algorithm for chemical equilibrium problems related to modeling of atmospheric organic aerosols. $\mathrm{J}$. Optim. Theory Appl., 130 (2006), No. 3, 375-407.

[5] H. Y. Benson, D. F. Shanno. Interior-point methods for nonconvex nonlinear programming: regularization and warmstarts. Comput. Optim. Appl., 40 (2008), No. 2, 143-189.

[6] A. Caboussat. Primal-dual interior-point method for thermodynamic gas-particle partitioning. Computational Optimization and Applications, 48 (2011), No. 3, 717-745.

[7] A. Caboussat, R. Glowinski. A numerical method for a non-smooth advection-diffusion problem arising in sand mechanics. Com. Pure. Appl. Anal, 8 (2008), No. 1, 161-178.

[8] A. Caboussat, R. Glowinski, V. Pons. An augmented Lagrangian approach to the numerical solution of a non-smooth eigenvalue problem. J. Numer. Math, 17 (2009), No. 1, 3-26.

[9] A. Caboussat, C. Landry, J. Rappaz. Optimization problem coupled with differential equations: A numerical algorithm mixing an interior-point method and event detection. J. Optim. Theory Appl., 147 (2010), No. 1, 141-156.

[10] G. R. Carmichael, L. K. Peters, T. Kitada. A second generation model for the regional-scale transport/chemistry/deposition. Atm. Env., 20 (1986), 173.

[11] E. J. Dean, R. Glowinski. An augmented Lagrangian approach to the numerical solution of the Dirichlet problem for the elliptic Monge-Ampère equation in two dimensions. Electronic Transactions in Numerical Analysis, 22 (2006), 71-96.

[12] E. J. Dean, R. Glowinski, G. Guidoboni. On the numerical simulation of Bingham viscoplastic flow: old and new results. Journal of Non Newtonian Fluid Mechanics, 142 (2007), 36-62.

[13] A. V. Fiacco, G. P. McCormick. Nonlinear programming : sequential unconstrained minimization techniques, Wiley, New York, 1968. 
[14] M. Fortin, R. Glowinski. Augmented Lagrangian Methods: Applications to the Numerical Solution of Boundary-Value Problems, Studies in Mathematics and Its Applications. Elsevier Science Ltd, 1983.

[15] R. Glowinski. Numerical Methods for Nonlinear Variational Problems, Springer-Verlag, New York, NY, 1984.

[16] R. Glowinski, P. Le Tallec. Augmented Lagrangians and Operator-Splitting Methods in Nonlinear Mechanics, SIAM, Philadelphia, 1989.

[17] J. Gondzio, A. Grothey. A new unblocking technique to warmstart interior point methods based on sensitivity analysis. SIAM Journal on Optimization, 19 (2008), No. 3, 1184-1210.

[18] M. Z. Jacobson. Fundamentals of Atmospheric Modeling, Cambridge, second edition, 2005.

[19] C. Landry. Numerical Analysis of Optimization-Constrained Differential Equations: Applications to Atmospheric Chemistry. PhD thesis, Ecole Polytechnique Fédérale de Lausanne, 2009. Available at http: / / library.epfl. ch/theses/?nr=4345.

[20] C. Landry, A. Caboussat, E. Hairer. Solving optimization-constrained differential equations with discontinuity points, with application to atmospheric chemistry. SIAM J. Sci. Comp., 31 (2009), No. 5, 3806-3826.

[21] D. Lanser, J. Verwer. Analysis of operator splitting for advection-diffusion-reaction problems from air pollution modelling. J. Comput. Appl. Math., 111 (1999), 201-216.

[22] C. M. McDonald, C. A. Floudas. GLOPEQ: A new computational tool for the phase and chemical equilibrium problem. Computers and Chemical Engineering, 21 (1996), No. 1, 123.

[23] G. J. McRae, W. R. Goodin, J. H. Seinfeld. Numerical solution of the atmospheric diffusion equation for chemically reacting flows. J. Comput. Phys., 45 (1982), No. 1, 1-42.

[24] Z. Meng, D. Dabdub, J. H. Seinfeld. Size-resolved and chemically resolved model of atmospheric aerosol dynamics. J. Geophys. Res., 103 (1998), 3419-3436.

[25] K. Nguyen, A. Caboussat, D. Dabdub. Mass conservative, positive definite integrator for atmospheric chemical dynamics. Atmos. Env., 43 (2009), No. 40, 6287-6295.

[26] K. Nguyen, D. Dabdub. Semi-lagrangian flux scheme for the solution of the aerosol condensation/evaporation equation. Aerosol Science \& Technology, 36 (2002), 407-418.

[27] R. T. Rockafellar. Convex analysis, Princeton University Press, Princeton, NJ, 1970.

[28] J. H. Seinfeld, S. N. Pandis. Atmospheric Chemistry and Physics: From Air Pollution to Climate Change, Wiley, New York, 1998. 
[29] S. Solomon, D. Qin, M. Manning, Z. Chen, M. Marquis, K.B. Averyt, M. Tignor, H.L. Miller, editors. Intergovernmental Panel on Climate Change: Fourth Assessment Report: Climate Change 2007, The Physical Science Basis, Cambridge University Press, 2007.

[30] B. Sportisse. A review of current issues in air pollution modeling and simulation. Comput. Geosci., 11 (2007), 159-181.

[31] J.G. Verwer, W. Hundsdorfer, J.G. Blom. Numerical time integration for air pollution models. Surveys Math. Ind., 10 (2002), 107-174.

[32] R. A. Zaveri, R. C. Easter, J. D. Fast, L. K. Peters. Model for simulating aerosol interactions and chemistry (MOSAIC). J. Geophys. Res. D (Atmospheres), 113 (2008), No. D13, D13204.

[33] R. A. Zaveri, R. C. Easter, L. K. Peters. A computationally efficient multicomponent equilibrium solver for aerosols (MESA). J. Geophys. Res. D (Atmospheres), 110 (2005), No. D24, D24203. 